\title{
Exposure and Factors Influencing the Progression of Diabetic Nephropathy AmongstType II Diabetes Mellitus Patients in Seremban, Negeri Sembilan
}

\author{
Wan Muhamad Amir W Ahmad ${ }^{1}$, Nor Azlida Aleng ${ }^{2}$, NurfadhlinaAbdul \\ Halim $^{3}$, MohdPouzi Hamzah ${ }^{4}$, NorizanMohamed ${ }^{5}$ andZalila Ali $^{6}$ \\ ${ }^{1,2,3,4,5}$ School of Informatics and Applied Mathematics Universiti Malaysia Terengganu (UMT) 21030 \\ Terengganu, Malaysia \\ ${ }^{6}$ School of Mathematics Sciences, UniversitiSains Malaysia (USM)11800 Minden, Pulau Pinang, Malaysia
}

\begin{abstract}
The case of diabetic nephropathy often arisesamong people with type 1 or type 2 diabetes. The incident of diabetic nephropathy has increased dramatically in the last decade. Increasing case of diabetic nephropathy had worried to most of diabetic patient. A cross-sectional study was conducted andfocused on two areas:modeling the applied logistic regression and todetermine the factors that influence diabetic nephropathy mellitus using structural equation modelling in Seremban, Negeri Sembilan.Three hundred eighty three diabetic patients were selected from Seremban. Of those who had diabetes, 172 (44.9\%) patients were female and 211 (55.1\%) were male.Finding from the previous research, the earliest age tostart diabetes mellitus is at 20 years old. Factors found to be significantly associated with diabetes mellitus on multivariate analysis werebeta blockers $(O R=3.942,95 \% C I=0.422,36.854)$, serum creatinine $(O R=1.057,95 \% C I=1.023,1.093)$, age $(O R=0.917,95 \% C I=0.821,0.97)$, blood glucose $(O R=1.564,95 \% C I=1.091,2.241)$, gender $(O R=20.396$, 95\% CI= 0.963, 431.892). In this paper, we found that age, being male,taking Beta-blockers having blood glucose level and serum creatininereported as influencing factors of type II diabetes mellitus.
\end{abstract}

Keywords: Logistic regression, SEM, diabetes mellitus, diabetic nephropathy.

\section{Introduction}

Diabetes mellitus (DM) type II is one of the major public health in the Asian-Pacific region due to the high medical and socioeconomic costs [1]. Diabetic nephropathy is much more common amongst diabetic patients. Previous research has been identified that diabetic patients have major risk factor for diabetic nephropathy.Based on the Diabetes Atlas, the highest number of people with diabetes would currently be in the European region with 48 million and Western Pacific Region with 43 million. However, by 2025, the greatest number of people with diabetes is expected to change in the South East Asia Region, with an estimated prevalence of $13.5 \%$ and in number with some 145 million people [2]. American Diabetes Association (ADA) examined the criteria for impaired fasting glycaemia (IFG) and recommended that the cut-point for IFG should be lowered to a fasting plasma glucose (FPG) $\geq 5.6 \mathrm{mmol} / \mathrm{L}(100 \mathrm{mg} / \mathrm{dl})$ [3].

A study in 2006, has estimated that for the urban population in China, the direct medical costs of diabetes are US\$45 per year for someone without complications, meanwhile for people with complications it will up to US\$1694 per year [4]. Although DM type I remain the most prevalent form of the disease, it is likely that DM type II will be the predominant form within 10 years. DM type II has already been reported in from Japan, Pacific Islands, Hong Kong, Singapore, China, Malaysia, Korea and Australia. Among children in Japan, it is accounting for $80 \%$ of childhood diabetes; the incidence has almost doubled between 1976-1980 and 19911995 [5].

This rising trend is due to many factors such as ethnicity, strong family history of DM type II, age, high body mass index (BMI) scores and central obesity indicators (waist-to-hip ratio and waist circumference), low birth weight, and "Westernization" (adoption of lifestyle habits characteristic of the more industrialized countries) [6].Second National Health and Morbidity reported that 3.4 million or 11.6\% Malaysians are diabetes sufferers in 2010. The Indians are leading in DM type II by $19.9 \%$, followed by Malays $11.9 \%$ and Chinese $11.4 \%$. Malaysia has the most number of overweight and obese people in Asia, obesity is a main cause of diabetes $54 \%$ of the adult population are either obese or overweight, compared to only $24.1 \% 10$ years ago.

High sugar intake (which also causes obesity) among Malaysians is one of the contributing factors to the high incidence of diabetes. Malaysians consume 26 teaspoons of sugar a day and are the eighth highest sugar users in the world.Amongst the 14 States in Malaysia, Negeri Sembilan, Malacca and Penang had the highest prevalence of DM type II of $15.3 \%, 15.2 \%$ and $14.9 \%$ respectively. The prevalence was higher in the urban at $12.2 \%$ (95\% CI: 11.6-12.7) compared to the rural areas at 10.6\% (95\% CI: 9.9-11.1) [7].DM can cause others serious illness and complications. Acute complications include diabetic ketoacidosis,nonketotic hyperosmolar 
coma, cardiovascular disease, chronic renal failure, and diabetic retinopathy (retinal damage). One of the common disease closely related to DM type II is diabetic nephropathy. Diabetic nephropathy has become the leading cause of end-stage kidney disease worldwide. Diabetic nephropathy is a progressive rise in urine albumin excretion, coupled with increasing blood pressure, leading to declining glomerular filtration and eventually end stage kidney failure [8]. In people with DM type II, the nephrons (small units in the kidney) thicken and slowly become scarred over time. Then, the kidneys begin to leak and protein (albumin) passes into the urine. People who have more severe and long-term (chronic) kidney disease may have symptoms such as fatigue most of the time, general ill feeling, headache, nausea and vomiting, poor appetite and swelling of the legs.

\subsection{Study Area}

\section{Material And Methods} Sembilan

This research was conducted in Seremban. Seremban is the capital of the Malaysian state of Negeri

\subsection{Study population}

We studied patient with Diabetes Mellitus Type II who attended the Hospital Seremban. A total 383 eligible patients were selected. They were more than 22 until 88 years old and diagnosed to have Diabetes Mellitus Type II. Some of them have been diagnosed to havediabetic nephropathy

Sample size calculation for the first objective:

The sample sizes required at analysis stage are as follows.

Anticipated population proportion $(p) \quad=0.057$

Level of significance

Absolute precision $(\Delta)$

$$
=5 \%(0.05)
$$

$= \pm 5 \%$

$$
\begin{aligned}
& =\left(\frac{1.96}{0.05}\right)^{2} 0.057(1-0.57) \\
& =82.59 \approx 83 \text { respondents. }
\end{aligned}
$$

According to Lwanga and Lemeshow, [7].

In this case the response rate is estimated at only $75 \%$, then add another $25 \%$ for data collection stage. So, we get $n=82.59+(0.25 \times 82.59)=103.2 \approx 103$. Samples of 103 respondents are required at the analysis stage. For this analysis, we used 103 respondents.

Sample size calculation for the second objective:

In this study, we calculate the sample size with manual calculation, with the significance level $(\alpha) 0.05$ and the power of study (1- $\beta$ ) of $80 \%$. The detectable hazard ratio of the presence of prognostic factor relative to absence of prognostic factors was decided by the researcher and expert opinion by clinicians [5]. Two Proportions formula is given as follows:

where:

$$
n=\frac{p_{0}\left(1-p_{0}\right)+p_{1}\left(1-p_{1}\right)}{\left(p_{0}-p_{1}\right)^{2}}\left(z_{\alpha}+z_{\beta}\right)^{2}
$$

$\mathrm{P}_{0}=$ Based on literature review, $\mathrm{P}_{1}=$ Based on expert opinion, $z_{\alpha}=z_{0.05}=1.9600$

(one tailed) and $z_{\beta}=z_{0.20}=0.8416$ (one tailed)

\begin{tabular}{l} 
Table 1. Sample Size Calculation \\
\begin{tabular}{llccccc}
\hline No & Objective & ${ }^{*} \mathrm{P}_{1}$ & $\mathrm{P}_{0}$ & Type 1 Error & Power & Sample Size \\
\hline 1 & $\begin{array}{l}\text { Patients withType II Diabetes Mellitus } \\
\text { having a diabetic nephropathy[4] } \\
\text { Calculation }\end{array}$ & 0.39 & 0.22 & $5 \%$ & $80 \%$ & 114 patients \\
& $n=\frac{0.39(1-0.39)+0.22(1-0.22)}{(0.39-0.22)^{2}} \times(1.96+0.80)^{2}=108 \approx 109$ patients & \\
\end{tabular} \\
\hline
\end{tabular}


After adding $10 \%$ estimated missing data, we get $n=109+(0.1 \times 109)=119.90 \approx 120$ per group, which can be obtained as follows:

i. Patients with Type II Diabetes Mellitus having a diabetic nephropathy

$=120$ patients

ii. Patients with Type II Diabetes Mellitus which does not hasa diabetic nephropathy

$=120$ patients

Therefore, a total patient to be sampled is $(120 \times 2)=240$ patients.

Table 2.Variable Description and Coding

\begin{tabular}{lll}
\hline \multicolumn{2}{l}{ Variable Name } & Variable Description and Coding \\
\hline 1. & Age & Age patient in years \\
2. & Sex & Patient gender $($ female $=0$, male $=1)$ \\
3. & Serum & Serum Creatinine \\
4. & Fasting Blood Glucose & Reading of level fasting blood glucose $(\mathrm{mg} / \mathrm{dL})$ \\
5. & Beta Blocker & Taking Beta Blocker $(0=$ no, $1=$ yes $)$ \\
6. & Diabetes Nephropathy & $(0=$ no, $1=$ yes $)$ \\
\hline
\end{tabular}

\section{Statistical Analysis}

The data were analyzed with SPSS software and SEM (Structural Equation Modeling). Multiple logistic regression analysis was employed to evaluate the associated factors and to determine all possible estimate risk. All $p$ values were two-tailed andthe level of significance was set at 0.25 based onMickey and Greenland (1989) [5].They recommend the alpha was set to 0.25 level and be used as a screening criterion for variable selection in logistic regression. Multiple logistic linear regression analysis with enter method was applied toDabetes Nephropathy data. We also applied structural equation modeling (SEM) to investigate and estimate causal relations using a combination of statistical data and qualitative causal assumptions, while controlling for the effects of other variables included in the model and adjusting for measurement errors.

\section{Results}

Logistic Regression Models Approach for Diabetes Mellitus

To explore the underlying association between diabetes mellitus nephropathy and the selected explanatory variables, a set of logistic regression models is fitted in this section. For the binary logistic regression approach we take the following dichotomous variables

$Y=0$, Diabetic and having diabetic nephropathy

$Y=1$, Diabetic with no diabetic nephropathy

Then we obtained the following model.

Table 3. Logistic regression model of the associated factors for nephropathy disease

\begin{tabular}{|c|c|c|c|c|c|c|}
\hline \multirow{2}{*}{$\begin{array}{l}\text { Dependent } \\
\text { Variable }\end{array}$} & \multirow[b]{2}{*}{ Independent Variable } & Std. Coefficient & \multirow[b]{2}{*}{ S.E } & \multirow{2}{*}{$\begin{array}{c}\text { Adjusted } \\
\text { OR* }^{*}\end{array}$} & \multicolumn{2}{|c|}{$\begin{array}{c}\text { 95\% CI for Adjusted } \\
\text { OR }\end{array}$} \\
\hline & & Beta $(\beta)$ & & & Lower & Upper \\
\hline \multirow{11}{*}{$\begin{array}{l}\text { Diabetic } \\
\text { Nephropathy } \\
\text { Disease }\end{array}$} & & & & & & \\
\hline & Demographic factors & & & & & \\
\hline & Age (in years) & -0.086 & 0.057 & 0.917 & 0.821 & 1.025 \\
\hline & Sex & -10.384 & 4.023 & 20.396 & 0.963 & 431.892 \\
\hline & Biochemical & & & & & \\
\hline & Serum Creatinine & 0.056 & 0.017 & 1.057 & 1.023 & 1.093 \\
\hline & Fasting Blood Glucose & 0.447 & 0.184 & 1.564 & 1.091 & 2.241 \\
\hline & Medication & & & & & \\
\hline & Beta Blocker & 1.372 & 1.141 & 3.942 & 0.422 & 36.854 \\
\hline & Hosmer and Lemeshow Test & $\mathrm{p}$-value 0.88 & & & & \\
\hline & Area under the Curve & $0.954(95 \% \mathrm{C}$ & $6,1.00)$ & & & \\
\hline
\end{tabular}

Table 3 shows the final model of logistic regression. In this multivariate analysis, only five variables appeared as significant factors $(\mathrm{p}<0.25)$. It is clearly observed from the results that age $(\mathrm{OR}=0.917,95 \% \mathrm{CI}=0.821$, 1.025), patient'ssex (OR $=20.4,95 \% \mathrm{CI}=0.963,431.9)$, has been negatively associated with the nephropathy 
diabetes.Serum Creatinine level $(\mathrm{OR}=1.057,95 \% \mathrm{CI}=1.023,1.093)$, betablocker $(\mathrm{OR}=3.942,95 \% \mathrm{CI}=0.422$, $36.854)$, and fasting blood glucose $(\mathrm{OR}=1.564,95 \% \mathrm{CI}=1.091,2.241)$ has been positively associated with nephropathy diabetes. Gardner and Altman (1989) [5] recommendation that 0.25 level be used as a screening criterion for variable selection in logistic regression. According to them, traditional level (such as 0.05) often fails to identify variables known to be important.

Diabetic nephropathy is the kidney disease that occurs as a results diabetes (Amir et al., 2012). From multiple logistic regression, we can see that among male patients, the risk of developing diabetic nephropathy among male patients is 20 times higher compared to female.For the patient whose taking a beta blocker as a medication. The risk of developing diabetic nephropathy among patients who consume beta blocker is 4 times higher compared to patients who do not take the beta blocker.This logistic regression model was fit based on a non-significant Lwanga and Lemeshow $[6,13]$ with $p=0.886$ and good area under the curve $(0.954)$.

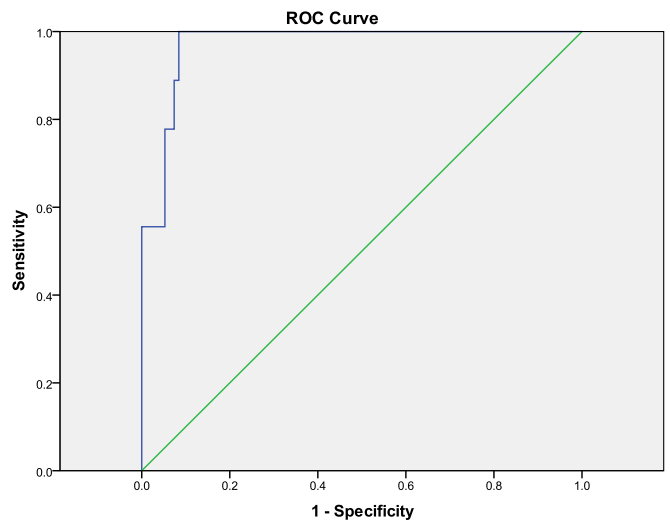

Figure.1 ROC Curve

We modelthe diabetic nephropathy case using structural equation modeling in order to determine or identify the relationship among variables. In the path diagram, kidney disease is predicted directly by sex, blood glucose, age and serum creatinine. In turn, sex is predicted by a beta blocker, and age is predicted by beta blocker and blood glucose. The path diagram also implies that the effect of beta blocker and fasting blood glucose on kidney disease are only indirect. They affect kidney disease only through their effects on sex, age and serum creatinine. Furthermore, some variables serve dual roles as predictors and outcome in the structural equation modeling diagram.

\section{Structural Equation Modeling}

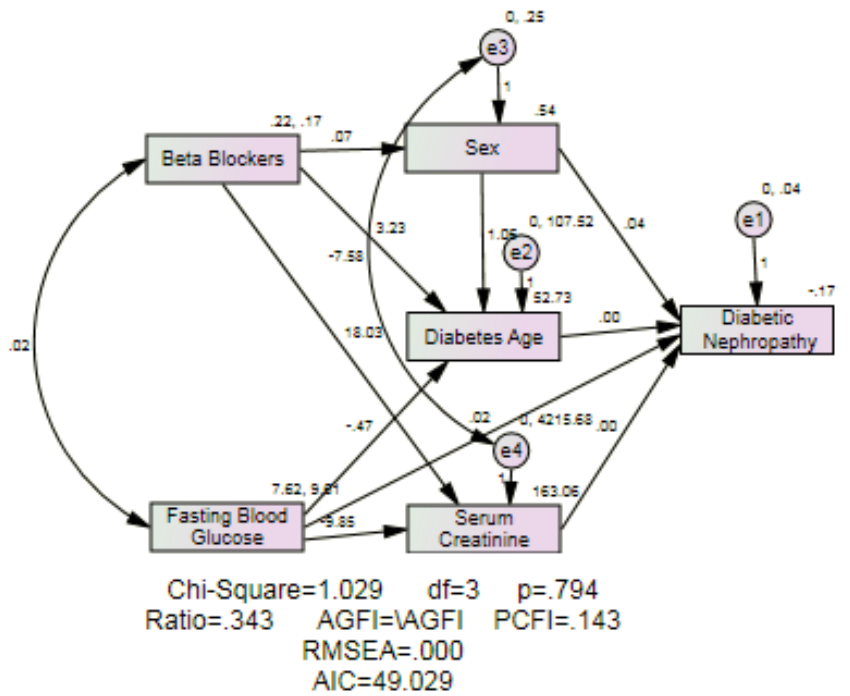

Figure 2. Structural equation modeling for diabetic nephropathy

\section{Serum Creatinine}

High serum creatinine level means the kidney is not well work. The normal measurement level of creatinine is 0.8 to $1.4 \mathrm{mg} / \mathrm{dL}$. Male has higher serotonin level compared to male because male usually have 
muscle mass.Blood test forcreatinine is a simplest way to monitor the kidney function. From the previous research, female has found to be lower creatinine $(0.6$ to $1.2 \mathrm{mg} / \mathrm{dL})$ than male, because they usually have less muscle mass creatinine based glomerular filtration rate is the best measure of kidney function since it account for age, bmianf sex. Usually, the measure of glomerular filtration rate declines when the kidney injured by disease.[Pradeep].The American Diabetes Association and the National Institutes of Health recommend Estimated glomerular filtration rate (eGFR) calculated from serum creatinine at least once a year in all people with diabetes for detection of kidney dysfunction.[Pradeep].Nayaket.al., (2011) have found that serum creatinine level area associated with type II diabetes. In their study, they found that diabetic over 50 years of age were found to have higher mean serum creatinine values than the non diabetics.

\section{Age and sex}

Structural equation modeling shows that age and sex have a direct effect on kidney disease. A number of studies in humans have shown that diabetes nephropathy increasing with age and sex. The incidence of progression of nondiabetic renal is generally larger found in men compared the woman. Many studies reported that the male sex is a risk factor for the development and rate progression of diabetic renal disease(Nayak).

\section{Beta Blocker}

Beta blocker has no direct effect upon kidney disease buat has an indirect effect through diabetes,sex and serum creatinine. The function of beta-Blockers is to slow the progression of diabetic nephropathy by lowering arterial pressure. Beta-blockers also block kidneys from producing a hormone called angiotensin II. In direct response to the action angiotensin, it will resulting in diabetic nephropathy. From the structural equation modeling we can see that there is a connection between blood glucose with beta blocker. The purpose of beta blockers is to reduce the deleterious effects on blood sugar levels. Usually among the diabetics,beta-blockers may cause higher blood sugar levels.

\section{Fasting Blood Glucose}

From Figure 1.4,we have found blood glucose that has no direct effect upon kidney disease but has an indirect effect through serum creatinine. Several studies have shown that the glucose associated serum creatinine.Recent research by Gualano (2011) has suggested that creatine may be an option in helping to maintain healthy blood sugar levels.Studies show that maintainbetter blood glucose can reduce the risk of getting diabetic nephropathy.

\section{Summary and Conclusion}

In this paper we examine the factors influencing the kidney disease. This paper provides an overviewof the problem associated with the relationship with kidney disease.Finding from the present study using multiple logistics show that there are five significant factors that influence kidney disease. They are age (OR $=0.917$, $95 \% \mathrm{CI}=0.821,1.025)$, patient'ssex $(\mathrm{OR}=20.4,95 \% \mathrm{CI}=0.963,431.9)$,Serum Creatinine level $(\mathrm{OR}=1.057$, $95 \% \mathrm{CI}=1.023,1.093)$, betablocker $(\mathrm{OR}=3.942,95 \% \mathrm{CI}=0.422,36.854)$, fasting blood glucose $(\mathrm{OR}=1.564$, $95 \% \mathrm{CI}=1.091,2.241)$ associated with nephropathy diabetes.

The results from structural equation modeling reveals that there four factors that have direct upon kidney disease. Two factors haveno direct effect upon kidney disease but has an indirect effect through serum creatinine, age and sex. Based on the goodness-of-fit indicesshown in Figure 2, it can be seen that $p$ value is not significant $p=0.794$ (accepting the null hypotheis) and therefore the ourmodel is well fit. The model fits the data well with $\chi^{2}=1.029$ withd.f $=3, p=0.794$. These two results are confirmed by both methods (multiple logistics regression and structural equation modeling).

We observed that diabetic nephropathy especially related to the type 2 diabetes. Pradeep (2010) point out that the diabetic nephropathy is the leading cause of chronic renal failure wordwide. It takes many years to develop in diabetic patients. Leaking of blood protein albumin into urine (microalbuminuria) cause the function of the kidney's filtering function begin to drop and resulting the body's retention of various waste. When a patient has developed the continual microalbuminuria, he or she possibly will be considered to have early sign for diabetic nephropathy. Early detection and taking an effective prevention step may help patient reverting to the standard level of microalbuminuria.

The process of kidney damage develops very slowly up to 15 to 25 years. The simplest way to monitor the kidney function byreading of urea nitrogen in blood and creatinine. Normal reading of blood urea nitrogen is around $7-20 \mathrm{mg} / \mathrm{dL}$.Screening for diabetic nephropathy and monitoring the function of kidney is the best way to detect diabetic nephropathy at the earliest stage. Detection of diabetic nephropathy at the earliest can possibly prevent progression to the end-stage. People with type I and type II diabetes are at risk of diabetic nephropathy. The diabetic nephropathy risk is higher if blood glucose is poorly controlled. Besides that, people with higher 
cholesterol level also have much more risk that others. According to Arora (2010), diabetes is the major cause of end-stage renal disease (ESRD).Traditional risk factor that is a strong relationship with ESRDareHypertension, hyperlipidemia and smoking. Albuminuria is a known as the best predictor for ESRD whilethe other factors as such serum albumin, serum creatinine and hemoglobin level enhancing the contribution of higher ESRD.

Evidence abounds shows that controlling the blood pressure, and controlling the level of glucose can reduce the risk of developing nephropathy. Common treatment of diabetic nephropathy ACE inhibitors and ARBs. Both oftwo types of treatment perform an action by delaying the progression of nephropathy. They react by slowing down the progress of microalbuminuria. Once a year screening for microalbuminuria level will help us to identify the cause of nephropathy at the early stage. Controlling blood glucose and periodically treatment for diabetes will improve or slow the rate of progression of diabetic nephropathy. (American Diabetes Association, 2004)

\section{References}

[1] Beatriz, A., Apodaca D.,Shah, E.,McCormack,V.,Cosío,F.G. \& Ruiz-Holguín, R. 2010.Prevalence of type 2 diabetes and impaired fasting glucose: cross-sectional study of multiethnic adult population at the United States-Mexico border, Rev PanamSaludPublica28(3).Pp 174-181.

[2] International Diabetes Federation. 2003. Diabetes Atlas. Executive Summary, $2^{\text {nd }}$ edition. Brussels: International Diabetes Federation.

[3] Genuth. S., Alberti, K.G., Bennett, P., Buse, J., Defronzo, R., Kahn, R., Kitzmiller, J., Knowler, W.C., Lebovitz, H., Lernmark, A., Nathan D., Palmer, J., Rizza., R, Saudek, C., Shaw, J., Steffes, M., Stern, M., Tuomilehto, J., Zimmet, P. 2003. Expert Committee on the Diagnosis and Classification of Diabetes Mellitus. Follow-up report on the diagnosis of diabetes mellitus.Diabetes Care; 26:3160-7.

[4] Shai I, Jiang R. Manson J.E, Stampfer M.J., Willett W.C., Colditz G.A.\& F.B, Hu. 2006. Ethnicity, obesity and risk of type 2 diabetes in women: a 20-year follow-up study. Diabetes Care. 29(7):1585-90.

[5] WHO. 2005. Type 2 Diabetes: Practical Targets and Treatments, Asian-Pacific Type 2 Diabetes Policy Group, Australia.

[6] Van Dam R.M. 2003.The epidemiology of lifestyle and risk for type 2 diabetes. Eur J Epidemiol.18(12):1115-25.

[7] Letchuman G. R., Wan Nazaimoon W.M., Wan Mohamad W.B., L.R.,Chandran, G.H. Tee, H.,Jamaiyah, M.R., Isa, H.,Zanariah, I.,Fatanah, Y.A. Faudzi. 2006. Prevalence of Diabetes in the Malaysian National Health Morbidity Survey III 2006, Med J Malaysia.Vol 65 No 3 September 2010.173-179.

[8] Enyiomaand Abdu Adem. 2005. Update in Diabetic Nephropathy.Int J Diabetes \& Metabolism 13: 1-9

[9] P.K. Dabla. 2010. Renal function in diabetic nephropathy.World J Diabetes. Vol 15; 1(2): 48-56

[10] Gualano, B. 2011. Creatine in Type 2 Diabetes: A Randomized, Double-Blind, Placebo-Controlled Trial Med Sci Sport

[11] American Diabetes Association. 2004. Nephropathy in Diabetes. Diabetes care.Vol 27.sepplement 1. pp 579-583.[20 February 2013]

[12] Nayak, B.S.,Butche , D.M., Bujhawan S., Chang D.,Chang S., Cabral-Samaroo D., CadanS., Buchoon V.,Budhram L., Boyce M., Teelucksingh S.(2011). Association of low serum creatinine, abnormal lipid profile, gender, age and ethnicity with type 2 diabetes mellitus inTrinidad and Tobago.Diabetes Research and Clinical Practical. Pp 342-347.

[13] Amir, W.M.A., NorAzlida, A. Zalila, A. \&Ariff, A. 2011. Modeling associated factors of HIV-infected tuberculosis (TB) patients using path model analysis. World Applied Sciences Journal 12(9): 1580-1584.

[14] Amir, W.M.A., Norizan, M., NorAzlida, \& A. Zalila. 2012. Influence of hypertension and diabetes Mellitus on family history of heart attack in male patients. Applied Mathematical sciences.6(66): 3259-3266. 